\title{
Variation der Präpositionswahl in direktiven Argumenten im Tschechischen und Deutschen kontrastiv
}

\section{Variation in preposition choice in directive arguments in Czech and German in contrast}

\author{
Agnes Kim, Maria Schinko
}

\begin{abstract}
Preposition choice is amongst the most prominent phenomena in which German in Austria differs from other varieties of German. Many instances are explained by language contact with Slavic languages in general and Czech in particular. This contribution sheds light on diamedial and diatopic variation of preposition choice in directive arguments (of the verbs Germ. gehen / Cz. jit/chodit) with the translation equivalents of school as the PPs inner objects. It chooses a contrastive, databased approach by analysing spoken and written corpora of various German varieties and Czech. We find evidence for diamedial variation in all analysed varieties or languages, respectively, with the prepositions Germ. in and Cz. do 'into' being relatively more frequent in spoken than in written language. Also, we identify two larger areal patterns with gradual transitions in Central Europe: first, a north-western one in the Hamburg/Hannover region, in which the preposition Germ. zu 'to' prevails; second a south-eastern one in Austria, Bavaria and the Czech Republic with the dominant preposition Germ. in / Cz. do 'into'.
\end{abstract}

\section{Keywords}

preposition choice; directive arguments; German; Czech; variation

Dieser Artikel ist im Rahmen des Teilprojekts „Deutsch und slawische Sprachen in Österreich: Aspekte des Sprachkontakts" (F 6006-G23, Projektleiter: Stefan Michael Newerkla) des vom Fonds für wissenschaftliche Förderung finanzierten SFB "Deutsch in Österreich: Variation - Kontakt - Perzeption" (F 60-G23) entstanden. Die Autorinnen danken außerdem Katharina Prochazka für ihre Unterstützung. 


\section{Präpositionswahl und Sprachkontakt in Zentraleuropa}

Die Wahl der Präposition in bestimmten Kontexten zählt zu denjenigen Phänomenen, die einerseits häufig als Spezifika von österreichischen Varietäten des Deutschen genannt werden (z. B. in De Cillia - Ransmayr 2019; Ebner 1988, 2008, 2009; Muhr 1995; Wiesinger 1996) bzw. sich durch korpusbasierte Forschung als solche erweisen (vgl. Ammon/ Bickel/Lenz 2016; Dürscheid/Elspaß/Ziegler 2018) und für die andererseits zahlreiche Beispiele als - eventuell - auf Sprachkontakt bzw. Konvergenz mit slawischen Sprachen auf Grund langfristiger Mehrsprachigkeit in der Habsburgermonarchie zurückgehend beschrieben werden (z. B. in Masař́k 1998; Newerkla 2007, 2009, 2013, 2017; Schuchardt 1884; Zeman 2003). Diese beiden Mengen sind zwar nicht ident, jedoch zu gewissen Teilen deckungsgleich. Beispielhaft seien (1-5) in Tabelle 1 angeführt.

Tabelle 1: Beispiele für sowohl als Austriazismen als auch als Kontaktphänomene beschriebene Präpositionalphrasen (PPn)

\begin{tabular}{|l|l|l|l|}
\hline & Deutsch in Österreich (DiÖ) & Deutsch in Deutschland (DiD) & Tschechisch \\
\hline (1) & $\begin{array}{l}\text { vergessen PAT[AKK] oder } \\
\text { PAT[auf + AKK] }\end{array}$ & vergessen PAT[AKK] & $\begin{array}{l}\text { zapomínat/zapomenout PA- } \\
\text { T[AKK] oder PAT[na + AKK] }\end{array}$ \\
\hline (2) & $\begin{array}{l}\text { sich erinnern/denken } \\
\text { PAT[an + AKK] oder PAT[auf + AKK] }\end{array}$ & $\begin{array}{l}\text { sich erinnern/denken } \\
\text { PAT[an + AKK] }\end{array}$ & $\begin{array}{l}\text { vzpomínat si/vzpomenout si/ } \\
\text { myslet PAT[na + AKK] }\end{array}$ \\
\hline (3) & auf Urlaub fahren/sein & in Urlaub fahren/sein & $\begin{array}{l}\text { jezdit na dovolenou/být na } \\
\text { dovolené }\end{array}$ \\
\hline (4) & $\begin{array}{l}\text { auf der Post sein/auf die Post } \\
\text { gehen }\end{array}$ & $\begin{array}{l}\text { bei der Post sein/zur Post } \\
\text { gehen }\end{array}$ & na poště/na poštu \\
\hline (5) & in der Nacht auf Sonntag & in der Nacht zum Sonntag & v noci na neděli \\
\hline
\end{tabular}

Die Relevanz von Aspekten der Präpositionswahl in der Untersuchung von slawischdeutschem Sprachkontakt in Österreich kann historisch, aber auch metalinguistisch begründet werden: Zunächst charakterisiert bereits Schuchardt (1884: 115) das „Gebiete [...] der Präpositionen “ als ein höchst variables und daher für Inter- und Transferenzen anfälliges $^{1}$ (vgl. auch Kim 2020: insbes. 115-117). Diese Bedeutung wird nicht nur in der Anzahl der jeweils für bestimmte Transferenzmuster stehenden Beispiele, die Schuchardt (1884: 115-119) selbst für Präpositionswahl bringt - nämlich 114 (vgl. Kim 2021)² - bestätigt, sondern auch dadurch, dass es sich um jene Phänomengruppe handelt, die - von allen bei Schuchardt identifizierbaren - auch bis ins 21. Jahrhundert hinein am

1 In Schuchardts (1884: 115) Wortlaut: „Kaum auf irgend einem Gebiete begeht der Fremde zahlreichere Fehltritte als auf dem der Präpositionen [...], und hier lässt sich der Einheimische um so leichter anstecken als ja auch für ihn der richtige Gebrauch derselben nicht immer leicht ist, und sie gern in synonymen und auch formell ähnlichen Verbindungen wechseln."

2 Die Differenz von 118 zu 114 Beispielen in den Zählungen von Kim (2020) und Kim (2021) ergeben sich durch unterschiedliche Bezugsgrößen: Kim (2020) zählt sämtliche von Schuchardt (1884: 115-119) genannten Beispielen, die hier genannte, auf Kim (2021) basierende Zählung hingegen berücksichtigt nur die Phänomengruppen „Präpositionswahl in der PP“ (101 Beispiele) und „Präpositionalargument anstatt von reinem Kasus“ (13 Beispiele), nicht jedoch die „Komparativkonstruktion“ (2 Beispiele) und die „Kontraktion von Präposition und Artikel“" (2 Beispiele). 
häufigsten behandelt wird. Von 15 bei Kim (2021) analysierten Werken nennen immerhin neun Beispiele für die Präpositionswahl betreffende Kontaktphänomene.

Die genannten Phänomene können nach zwei Gesichtspunkten klassifiziert werden, nämlich (a) nach dem Muster der Variation innerhalb der deutschen Varietäten und (b) nach der syntaktischen Position der betreffenden Präpositionalphrase (PP). Nach ersterem Kriterium können primär solche Phänomene, die Aspekte der Rektion eines spezifischen Verbs betreffen (z. B. [1]; vgl. Kim - Scharf - Šimko 2020) von solchen der Präpositionswahl im eigentlichen Sinn unterschieden werden, die - auszugsweise - im vorliegenden Artikel nähere Behandlung finden.

In Abschnitt 2 wird eine Einteilung der PPn nach dem zweiten Kriterium skizziert werden, wobei spezifisch österreichisch-deutsche, angebliche Kontaktphänomene im Bereich der Adverbiale selten sind und nur einige wenige temporale betreffen (z. B. [5]). Deutlich häufiger haben die betreffenden Phänomene den Status von direktiven/lokalen bzw. Präpositionalargumenten (z. B. [2-4]).

Dieser Artikel beschränkt sich auf direktive/lokale Präpositionalargumente. In ihnen ist, wie in Abschnitt 2 herausgearbeitet wird, die Präpositionswahl nicht primär an das

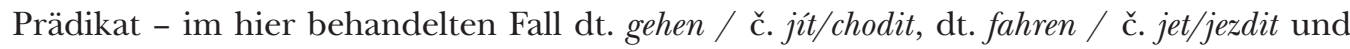
dt. unterrichten / č. učit/naučit (vgl. Abschnitt 3, Punkt c) - gebunden, sondern auch durch die regionskonstituierende Semantik der gewählten Präposition motiviert. Vor diesem Hintergrund stellen wir die übergeordnete Frage, ob - bzw. wenn ja, in welchen Kontexten - sich die regionskonstituierende semantische Komponente von bestimmten räumlichen Präpositionen im DiÖ von jener ihrer Entsprechungen im DiD unterscheidet und ob sie sich in diesen Fällen mit jenen ihrer tschechischen (Quasi-)Äquivalente deckt. Grundvoraussetzung und Ziel dieses Beitrags ist jedoch, zu klären, welche diatopischen, diamedialen und diachronen Variationsmuster in Bezug auf die Präpositionswahl im deutschen Sprachraum bestehen und ob bzw. wie sich das Tschechische (und andere slawische Sprachen) zu diesen verhalten. ${ }^{3}$ Dazu wird ein kontrastiver, datenbasierter Ansatz gewählt, wobei überwiegend PPn mit dem Lemma dt. Schule / č. škola (vgl. Abschnitt 3, Punkt c) sowie - zu Kontrastzwecken -mit dt. Post / č. pošta (vgl. Abschnitt 7) berücksichtigt werden.

\section{Präpositionswahl in direktionalen und lokalen Argumenten}

Wie in Bezug auf jede Wortart unterscheidet sich die Definition der Wortart Präposition entsprechend der einer Arbeit zugrunde gelegten Theorie, wobei Grießhaber (2007: 634) zufolge meist ein „Mischverfahren von zunächst morphologischen und syntaktischen Kriterien unter Berücksichtigung semantischer Aspekte“ angewandt wird. Für die vorliegende Untersuchung sind insbesondere Syntax und Semantik von Präpositionen von Relevanz, weshalb diese näher behandelt werden, während andere Eigenschaften

3 Für den deutschen Sprachraum liegen korpus- oder befragungsbasierte Kartierungen zu einzelnen Phrasen in der Variantengrammatik (Dürscheid - Elspaß - Ziegler 2018; z. B. zu auf die/zur Post, auf/in Urlaub etc.) sowie im Atlas zur deutschen Alltagssprache (Elspaß - Möller 2003ff.; z. B. zu auf/in/an der Schule/dem Gymnasium) vor. 
ausgespart bleiben (vgl. hierfür den Überblick in Grießhaber 2007). Dabei beschränken wir uns im Einklang mit dem Gegenstand des Artikels auf die Lokalisierungsfunktion primärer Präpositionen und lassen andere, z. B. temporale oder kausale Bedeutungen sowie sekundäre oder tertiäre Präpositionen außer Acht.

Syntaktisch definieren Zifonun - Hoffmann - Strecker (1997: 44-45) Präpositionen als Funktoren (oder: operative Terme), die durch die Expansion nominaler Phrasen PPn bilden und dabei den Kasus der expandierten Phrase regieren. Zusätzlich stellen sie eine semantisch spezifizierte Relation zwischen zwei Argumenten her, wobei das sogenannte innere Argument durch die von der Präposition regierte Phrase bezeichnet wird, wohingegen das äußere Argument in der syntaktisch übergeordneten Struktur zu finden ist (vgl. Zifonun - Hoffmann - Strecker 1997: 2099-2100). In beiden unter (6) verzeichneten Beispielen ist demnach das Regal das innere Argument, in Bezug auf das durch die Präposition eine Region spezifiziert wird (Bedeutungskomponente $a$ ). Zu dieser Region des inneren Arguments wird das äußere Argument - die Katze - in Beziehung gesetzt (Bedeutungskomponente $b$ ).
a) Die Katze
schläft
$\mathrm{PP}[$ in
dem Regal]. ÄUSSERES ARG. INNERES ARG.
b) Die Katze ÄUSSERES ARG.
$\operatorname{PP}[$ in
dem Regal]
schläft. INNERES ARG.

Welche der beiden Bedeutungskomponenten aktiviert sind, entscheidet die syntaktische Position der PP: Während in adverbialen oder attributiven Verwendungen einer PP beide dieser Komponenten für die Präpositionsselektion von Relevanz sind, übernimmt im Fall, dass es sich bei der PP um ein direktionales oder lokales Argument eines Bewegungs- oder Positionsverbs handelt, das Verb Bedeutungskomponente $b$ : Dass sich die Katze auf ein Ziel zubewegt, wird in Beispiel (7a) durch das Verb ausgedrückt, ebenso wie die Tatsache, dass die Katze an einem bestimmen Ort sitzt, in Beispiel (7b). Die Präposition erfüllt nur noch den Ausdruck von Bedeutungskomponente $a$, indem sie die Region des Zielobjekts bzw. Orts spezifiziert und damit ausdrückt, dass die Katze in das Regal hineinläuft (etwa, um sich zu verstecken und nicht nur auf es zu bzw. in ihm) und nicht auf ihm sitzt.

a) Die Katze läuft PP[in das Regal].

b) Die Katze sitzt PP[in dem Regal].

Die beiden unter (7) angeführten Beispiele drücken - durch das Verb kodiert - zwei verschiedene Formen der Relation aus. Während im Fall von (7a) sowie bei anderen Bewegungsverben die Relation emergent, d. h. im Entstehen begriffen ist, existiert sie in (7b) bereits (vgl. Leys 1989). Dies hat - sofern sie auf Grund ihrer Bedeutungskomponente $a$ selegiert werden - Auswirkungen auf die Kasusrektion der sogenannten Wechselpräpositionen an, auf, in, hinter, neben, über, unter, vor und zwischen, die allesamt primär räumliche Relationen ausdrücken (vgl. Wöllstein - Dudenredaktion 2016: 620; Zifonun 
- Hoffmann - Strecker 1997: 2105). In Bezug auf emergente Relationen regieren sie den Akkusativ, in Bezug auf existente hingegen den Dativ.

Die vorliegende Untersuchung bezieht sich primär auf direktive, sekundär auch auf lokale Argumente und Adverbiale. Um Vollständigkeit zu gewährleisten, sei jedoch noch die dritte mögliche syntaktische Position von PPn erwähnt, jene als Präpositionalargumente (8). In diesen Fällen ist die Präposition semantisch nahezu komplett leer und erfüllt fast ausschließlich syntaktische Funktionen (Zifonun - Hoffmann - Strecker 1997: 2155), wobei den Grammatikalisierungsprozessen eine lokale Relation zugrunde liegen kann, wie sie in Beispiel (8) noch greifbar ist (vgl. auch Hundt 2001).

Er übersetzt hauptsächlich Gesetzestexte PP ins Tschechische].

Im Fall solcher Präpositionalargumente ist die Selektion der Präposition an das Verb und seinen Valenzrahmen gebunden, was sich darin ausdrückt, dass sie nur durch W-Präpositionaladverbien (z. B. worin?) oder PPn (z. B. in welche Sprache?) erfragt werden können. Darin unterscheiden sie sich von den direktionalen und lokalen Argumenten, die auf die Fragen wohin? oder wo? antworten. Es stellt sich die Frage, wodurch die Präpositionsselektion in direktionalen und lokalen Argumenten gesteuert wird.

Grießhaber (1999: 88-107) entwickelt im Rahmen der funktionalen Pragmatik einen Algorithmus zur Wahl einer bestimmten Präposition in statischen (lokalen), direktiven und ursprungsbestimmten PPn, in dem neben diesen elementaren Relationen (1. Schritt) auch die Klassifikation des inneren Arguments (2. Schritt) sowie die komplexe lokale Relation dessen zum äußeren Argument (3. Schritt) bestimmend sind. Die inneren Argumente einer PP werden ihm zufolge entweder in Objekte des Wahrnehmungsoder Vorstellungsraumes eingeteilt, wobei erstere nach „wahrnehmbaren Merkmalen“ als Punkt, Raum, Fläche oder Person subklassifiziert werden (Grießhaber 1999: 97) und bei letzteren die Subklassifikation in Bezirk, Amt, Aktant, Siedlung nicht unmittelbar von der „sinnlich wahrnehmbaren Form“ gesteuert wird (Grießhaber 1999: 98). Zusätzlich ist im Deutschen auch die komplexe lokale Relation, deren entscheidendes Kriterium die Distanz des inneren zum äußeren Argument darstellt, von Relevanz für die Präpositionswahl. Grießhaber (1999: 103) unterscheidet zwischen Inklusions- und Konnexbeziehung, jener der engeren und der offenen Region sowie der Umgebungsbeziehung. Tabelle 2 zeigt die Präpositionen im Deutschen, die in der vorliegenden Untersuchung von Relevanz sind, sowie ihre tschechischen Äquivalente. In den ersten beiden Spalten wird zusätzlich angegeben, für welche Argumentklasse und welche komplexe Relation Grießhaber (1999: 107) die deutschen Präpositionen als typisch erachtet. Dabei werden nur solche berücksichtigt, in die das untersuchte Lemma Schule (vgl. Abschnitt 3, Punkt c) potentiell eingeordnet werden könnte. 
Tabelle 2: Relevante Präpositionen in direktiven und lokalen PPn mit typischer Argumentklasse und komplexer Relation nach Grießhaber (1999)

\begin{tabular}{|c|c|c|c|c|c|}
\hline \multirow[b]{2}{*}{$\begin{array}{l}\text { Argu- } \\
\text { ment-klasse }\end{array}$} & \multirow[b]{2}{*}{$\begin{array}{l}\text { komplexe Re- } \\
\text { lation }\end{array}$} & \multicolumn{2}{|c|}{ Direktive PPn } & \multicolumn{2}{|c|}{ Lokale PPn } \\
\hline & & Deutsch & Tschechisch & Deutsch & Tschechisch \\
\hline Raum/Bezirk & Inklusion & in + AKK & do + GEN & in + DAT & $v+$ LOK \\
\hline $\begin{array}{l}\text { Raum/Bezirk } \\
\text { Fläche/Amt }\end{array}$ & offene Reg. & $z u+D A T$ & $k+\mathrm{DAT}$ & bei + DAT & $u+$ GEN \\
\hline Fläche/Amt & Konnex & auf + AKK & \multirow[b]{2}{*}{$n a+\mathrm{AKK}^{4}$} & auf + DAT & \multirow[b]{2}{*}{$n a+$ LOK } \\
\hline $\begin{array}{l}\text { Raum/Bezirk } \\
\text { Fläche/Amt }\end{array}$ & enge Reg. & $a n+\mathrm{AKK}$ & & $a n+D A T$ & \\
\hline
\end{tabular}

\section{Methodischer Zugang}

Um der Beantwortung der Fragestellung gerecht zu werden, wurde ein (a) in zweierlei Hinsicht kontrastiver, (b) korpuslinguistischer und (c) lemmabasierter Ansatz gewählt.

Ad. (a): Dabei wurden die Sprachen Deutsch und Tschechisch verglichen, um sowohl Aspekte des Sprachkontaktes als auch der arealen Konvergenz berücksichtigen zu können. Weiters wurden sowohl das Deutsche in Österreich (DiÖ) als auch das Deutsche in Deutschland (DiD) sowie gesprochene und geschriebene Sprachdaten aus allen drei Sprachen bzw. Varietäten untersucht, um Aspekte der horizontalen (arealen) und vertikalen Variation miteinbeziehen zu können.

Ad. (b): Als Ausgangskorpus diente dabei das zwischen 2016 und 2019 von den Teilprojekten PP03 und PP08 erstellte Korpus des Spezialforschungsbereichs ,Deutsch in Österreich: Variation - Kontakt - Perzeption' (vgl. Koppensteiner - Lenz 2017; Lenz [et al.] 2019; i. d. F. SFB-Korpus), welches gesprochene Sprachdaten aus 13 österreichischen Orten verschiedener Dialektregionen beinhaltet. In formellen Interviews wurden standardnähere, in Freundesgesprächen dialektalere Varietäten erhoben. Das Korpus wird mit dem Ende der Projektlaufzeit (2023) im Rahmen der Forschungsplattform DiÖ-KORP öffentlich zugänglich sein. Als Quelle für die gesprochenen Sprachdaten zum DiD fungierte das Korpus Deutsche Umgangssprachen (vgl. Pfeffer 1975; IDS 2015; i. d. F. Pfeffer-Korpus) aus dem Jahr 1961, bestehend aus Berichten, Erzählungen und Vorträgen, das in der Datenbank für Gesprochenes Deutsch (DGD) des Leibniz-Instituts für Deutsche Sprache (IDS) in Mannheim zugänglich ist. ${ }^{5}$ Daten zum gesprochenen

4 Zur (Quasi-)Äquivalenz von dt. auf/an und č. na vgl. Bednarský (2002). Aus pragmatischen und darstellungstechnischen Gründen werden für die Analysen und Darstellungen in diesem Artikel dt. auf und č. na zu einer Kategorie zusammengefasst, dt. an wird jedoch separat ausgewertet.

5 Die Wahl des Pfefferkorpus als Vergleichskorpus für die „Umgangssprache“ des DiD erfolgte auf Grund der Tatsache, dass keine mit den Korpora für das DiÖ und das Tschechische vergleichbaren, rezenteren Sprachdaten frei zugänglich sind. Das zwischen 2006 und 2009 erhobene Korpus des Projekts „Deutsch heute“ (IDS 2021; vgl. Kleiner 2015; Kleiner 2011-2021) ist zwar über die DGD frei zugänglich, enthält jedoch nur standardnahe Varietäten von ausschließlich männlichen Sprechern. Die Daten des zwischen 2008 und 2012 durchgeführten Projekts „Sprachvariation in Norddeutschland“ (SiN; vgl. Elmentaler [et. al.] 2015) hingegen wurden primär in Form des Norddeutschen Sprachatlas (NOSA; vgl. Elmentaler - Rosenberg 2015) aufbereitet 
Tschechischen lieferte das zwischen 2012 und 2017 erstellte Korpus ORTOFON (v1) des Český národní korpus (ČNK), welches spontane, in informellen Situationen gebrauchte gesprochene Sprache umfasst (vgl. Kopřivová [et. al.] 2017; Komrsková [et. al.] 2017).

Die Analyse der geschriebenen Varietäten des Deutschen basiert auf der morphosyntaktisch mit dem TreeTagger annotierten Version 2014-I des Deutschen Referenzkorpus (DeReKo, TAGGED-T2; vgl. IDS 2014), das ebenfalls vom IDS Mannheim bereitgestellt wird. Dieses umfasst Zeitungstexte aus Österreich, Deutschland und der Schweiz aus den Jahren 2010 bis 2014. Als synchrones Korpus des geschriebenen Tschechischen diente - um Vergleichbarkeit zu gewährleisten - ein nur aus publizistischen Texten derselben Zeitspanne bestehendes Subkorpus des SYNv7 des ČNK (vgl. Křen [et. al.] 2018; Hnátková [et. al.] 2014). Bei der Auswahl der Korpora geschriebener Sprache wurde auf das Vorhandensein morphosyntaktischer Annotationen Wert gelegt, da durch diese auch ohne manuelle Annotation jeder einzelnen Konkordanz Erkenntnisse gewonnen werden konnten. Manuelle Annotation war daher nur bei den Korpora der gesprochenen Sprache notwendig.

Für eine detaillierte Analyse der geschriebenen Varietäten beider Sprachen, die auch auf Grund der anzunehmenden Quote an falschen Treffern in den nur quantitativ, nicht aber qualitativ ausgewerteten Korpora angezeigt war, wurde allerdings ein Subkorpus aus dem DeReKo (Version 2014-I, TAGGED-T2) sowie den publizistischen Texten aus dem SYN2015 (vgl. Křen [et. al.] 2015; Křen [et. al.] 2016) ${ }^{6}$ erstellt, wobei der Fokus bei den deutschsprachigen Varietäten - um kontrastive Aspekte zu berücksichtigen - auf Ostösterreich (Niederösterreichische Nachrichten und Burgenländische Volkszeitung) und Nordwestdeutschland (Hamburger Morgenpost und Hannoversche Allgemeine) lag. Die Konkordanzen aus diesen Korpora wurden anschließend ebenfalls manuell annotiert.

Hinweise auf etwaige historische (Dis-)Kontinuitäten im ostösterreichischen Raum lieferte abschließend außerdem ein kleines diachrones Korpus bestehend aus im ANNO (AustriaN Newspapers Online, https://anno.onb.ac.at), dem digitalen Zeitschriftenlesesaal der Österreichischen Nationalbibliothek verfügbaren Zeitungsartikeln der Jahre 1778-1836 aus Wien.

Ad. (c): Der Fokus der Analyse lag auf dem in den verwendeten gesprochenen Korpora frequentesten Lemma in direktionalen PPn, das unter den vermeintlichen Kontaktphänomenen des Deutschen und Tschechischen aufgelistet war, nämlich dt. Schule bzw. č. škola. In den umfangreicheren geschriebensprachlichen Korpora wurde es in direktiven Phrasen ausschließlich in Kombination mit den Verben dt. gehen bzw. č. jit/chodit

und sind nicht in Form eines Korpus verfügbar. Außerdem beschränken sie sich auf den norddeutschen Raum und weibliche Sprecherinnen. Die Aufnahmen, die im Rahmen des seit 2008 finanzierten Langzeitprojekts Regionalsprache.de (REDE; vgl. Ganswindt - Kehrein - Lameli 2015) mit männlichen Sprechern aus ganz Deutschland in verschiedenen Sprachlagen gemacht wurden, sind nur in Form von Audiodateien, nicht aber transkribiert auf der Plattform des Projekts (www.regionalsprache.de, zuletzt geprüft am 26. 06. 2021) zugänglich.

$6 \quad$ SYNv7 und SYN2015 unterscheiden sich dadurch, dass letzteres Korpus ein nach Textsorten balanciertes Referenzkorpus ist, während es sich bei ersterem um ein nicht-balanciertes, versioniertes Korpus handelt, in dem demnach publizistische Texte die belletristischen und die Fachtexte bei weitem überwiegen. Daher unterscheiden sie sich auch in der Größe (SYNv7: rund 4255 Mill. Token, SYN2015: rund 100 Mill. Token). 
und dt. fahren bzw. č. jet/jezdit abgefragt. Zur Vergleichbarkeit mit lokalen Präpositionalsätzen wurde mittels Kookurrenzanalysen das Verb dt. unterrichten bzw. č. učit/naučit als das häufigste identifiziert, wobei bei der Interpretation der tschechischen Daten zu beachten ist, dass dieses aus technischen Gründen nicht vom reflexiven Lemma učit se/ naucit se ,unterrichten' unterschieden werden kann. Die folgende Argumentation konzentriert sich jedoch primär auf direktive Kontexte. In der Auswertung wurden nur die in Tabelle 2 dargestellten Präpositionen berücksichtigt.

\section{Gesprochene Varietäten im Fokus}

Abb. 1 gibt einen Überblick über die Präpositionswahl in den gesprochensprachlichen Korpora und zeigt, dass in diesen in direktionalen PPn im Tschechischen, Deutschen in Österreich (DiÖ) ${ }^{7}$ und Deutschen in Deutschland (DiD) die Präposition dt. in / č. do dominiert. Im Tschechischen wird sie in $92 \%$, im DiÖ in $81 \%$ der Fälle gebraucht. Im DiD kommt sie in nur 52,2 \% der Fälle zur Anwendung. Die Präposition dt. $z u / \check{c}$. $k$ ist im tschechischen Korpus nicht vertreten, im DiÖ kommt sie in $4 \%$, im DiD hingegen in $37 \%$ der Fälle vor. Die Präpositionen dt. auf/an / č. na machen im Tschechischen 8 \%, im DiÖ $2 \%$ und im DiD $7 \%$ aus. Charakteristisch für das DiÖ - und ausschließlich für das DiÖ - ist die Elision, also das Weglassen der Präposition (8), die mit 13 \% zweithäufigste Variante im SFB-Korpus.

(SFB-Korpus, WEI-m-o-0056)

schui gongen bin i eintlich nia so richtig gean.

Schule gegangen bin ich eigentlich nie so richtig gern.
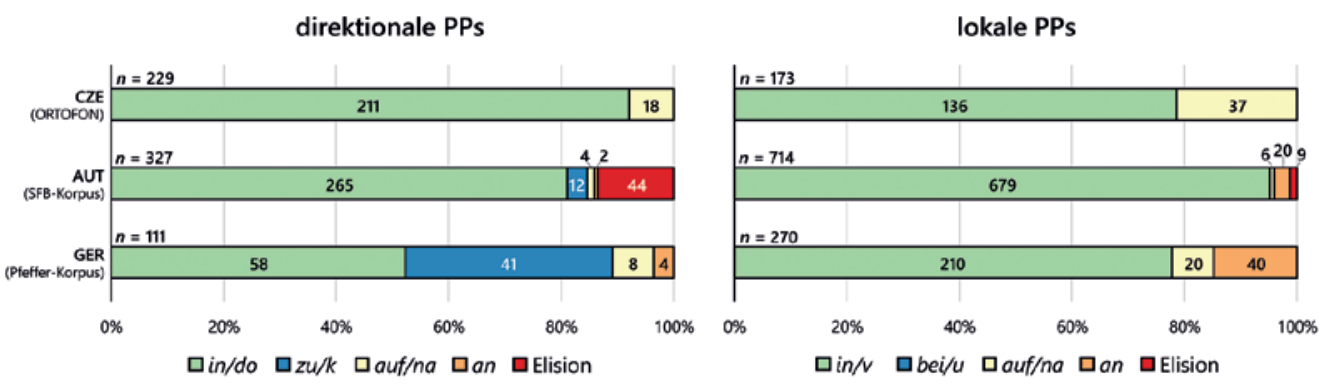

Abb. 1: Direktionale und lokale PPn aus den gesprochensprachlichen Korpora - Überblick

In lokalen PPn überwiegt die Präposition dt. in / č. $v$ ebenfalls, wobei sie im Tschechischen in $78 \%$ der Fälle und somit seltener als in direktiven Kontexten gebraucht wird.

7 Die Belege für das DiÖ stammen in dieser Auswertung ausschließlich aus dem SFB-Korpus. 
In den beiden deutschsprachigen Varietäten ist sie hingegen häufiger. Die Präposition dt. bei / č. $u$ - als lokale Entsprechung der direktiven Präposition dt. $z u$ / ̌́. $k$ - kommt im Korpus nicht vor. Dt. auf / ̌̌. na werden auch in direktionalen Kontexten vorwiegend im Tschechischen $(21 \%)$ und im DiD $(7 \%)$ verwendet. Im Vergleich zu den direktiven PPn kommt der Gebrauch der Präposition dt. an im DiÖ auf $3 \%$ und im DiD auf $15 \%$. Diese Häufigkeit könnte stark vom Lemma abhängen. Die Elision der Präposition im DiÖ ist auf direktive Kontexte beschränkt.

Die Daten zum Deutschen lassen eine areale Variation über den gesamten deutschen Sprachraum hinweg vermuten, die mithilfe (sprach-)kartographischer Verfahren basierend auf den sowohl im Pfeffer-Korpus als auch im SFB-Korpus zu den Erhebungsregionen bzw. -orten enthaltenen Daten bestätigt wird (vgl. Abb. 2, 3). Der Fokus liegt dabei auf den direktionalen PPn, welche eine höhere Variation aufweisen. Für das bundesdeutsche Gebiet gilt, dass die Präposition dt. $z u$ die dominante Variante in Nordwestdeutschland ist, im Südosten hingegen kaum vorkommt. In den südlichen Bundesländern und dabei insbesondere im südöstlichsten Bayern überwiegt dt. in. Im österreichischen Gebiet wird dt. in in fast allen Erhebungsorten des SFB-Korpus am häufigsten verwendet. Aufmerksamkeit verdient hier die regionale Verteilung der Elision der Präposition. Diese wird im südbairischen Gebiet mit dem Epizentrum Kärnten durchgängig verwendet und reicht bis nach Tarrenz in Tirol. In Weißbriach in Kärnten stellt sie sogar die häufigste Variante dar. All diese Ergebnisse sind aber nur bedingt aussagekräftig und pauschalisierbar, da die Anzahl der Belege pro Bundesland bzw. Erhebungsort höchst unterschiedlich ist. Im Pfeffer-Korpus ist die Region außerdem nicht für alle Belege spezifiziert. Dennoch sind erste Tendenzen in Bezug auf die areale Verteilung der Präpositionen erkennbar.

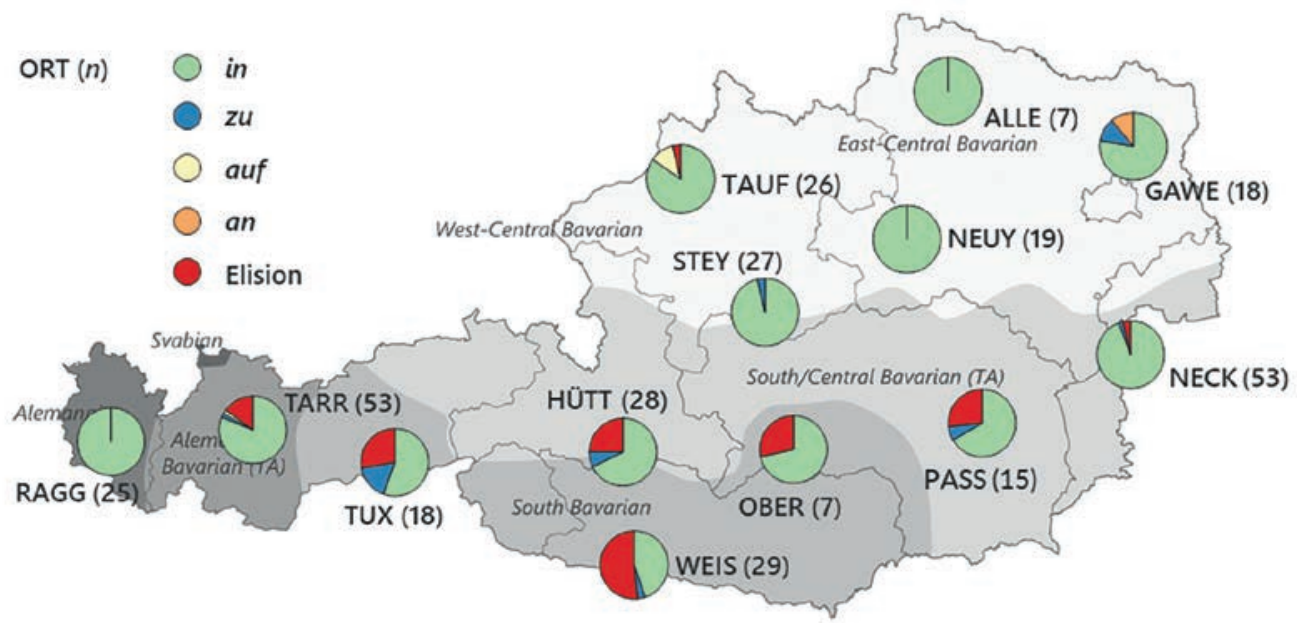

Ortkürzel (von Westen nach Osten): RAGG ... Raggal, TARR ... Tarrenz, TUX ... Tux in Tirol, HUTT ... Hüttschlag. WEIS ... Weißbriach, TAUF ... Taufkirchen, STEY ... Steyerling, OBER ... Oberwölz, NEUY ... Neumarkt an der Ybbs, ALLE ... Allentsteig, PASS ... Passail, GAWE ... Gaweinstal, NECK... Neckenmarkt

Abb. 2: Direktionale PPn aus dem Pfeffer-Korpus mit Schule als innerem Argument 


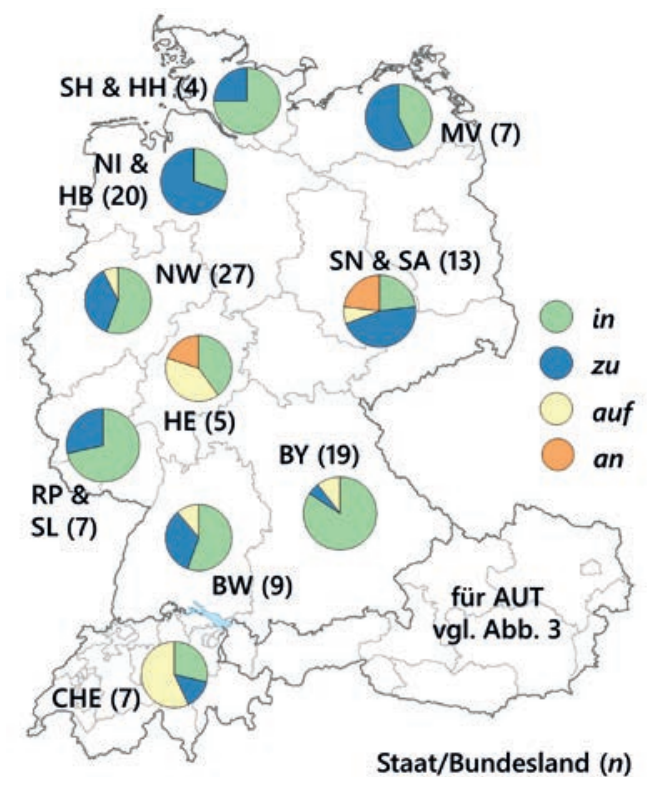

Abb. 3: Direktionale PPn aus dem SFB-Korpus mit Schule als innerem Argument

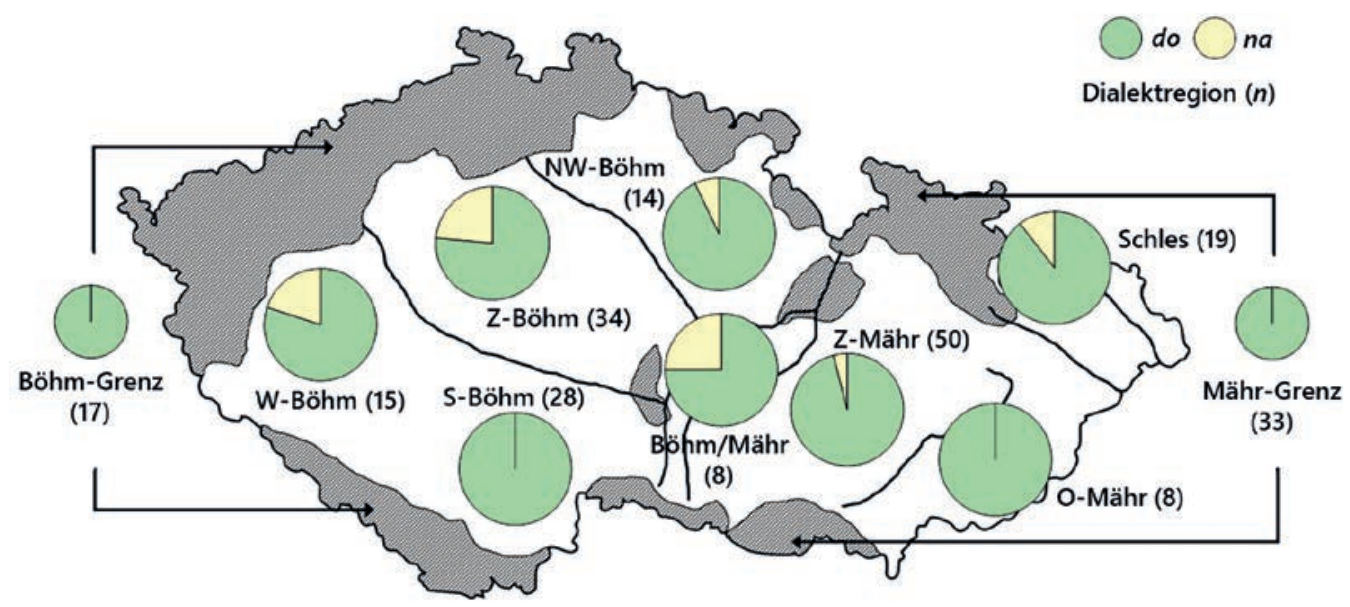

Abb. 4: Direktionale PPn aus dem ORTOFON mit škola als innerem Argument; Grundkarte nach Kloferová (2017)

Auch für das tschechische Sprachgebiet ist eine Auswertung nach Dialektregionen möglich (vgl. Abb. 4). Dabei zeigt sich, dass die Präposition č. do über den gesamten tschechischen Sprachraum dominiert. Der Gebrauch von č. na konzentriert sich auf Böhmen, wobei es in Aufnahmen aus dem zentral- und westböhmischen sowie dem 
böhmisch-mährischen Übergangsgebiet in rund $25 \%$ der Fälle gebraucht wird - deutlich häufiger als im gesamten tschechischen Sprachraum (8 \%). Auffallend ist außerdem, dass die Präposition č. $n a$ weder in den böhmischen noch in den mährischen Grenzregionen, die an den deutschen Sprachraum angrenzen, vorkommt. Zu überprüfen, ob dieses Variationsmuster tatsächlich diatopisch bedingt ist und nicht primär anderen Einflussfaktoren - etwa semantischen wie jenem des Schultyps - unterliegt, muss in künftigen Studien eruiert werden.

Zusammenfassend können für die gesprochenen Korpora in direktiven Kontexten sowohl sprachenübergreifend als auch innerhalb der deutschsprachigen Varietäten einerseits Gemeinsamkeiten, andererseits auch Differenzen in Bezug auf die Präpositionswahl identifiziert werden. Das DiÖ und das Tschechische verbindet die Dominanz der quasiäquivalenten Präpositionen dt. in / č. $d o$, welche auch in den südlichen Bundesländern des DiD und vor allem in Bayern überwiegt. Dt. auf / č. $n a$ werden sowohl in der Schweiz als auch in Mitteldeutschland sowie im Tschechischen - insbesondere in Böhmen - verwendet. In Mitteldeutschland tritt außerdem die Präposition dt. an vermehrt auf. Die Präposition dt. zu dominiert im Nordwesten Deutschlands; die Elision der Präposition ist hingegen auf das DiÖ, genauer auf die südbairische Dialektregion beschränkt.

\section{Geschriebene Varietäten im Fokus}

Durch ihre morphosyntaktische Annotation war es möglich, die geschriebenen Korpora zur Gänze nach PPn mit dem Lemma dt. Schule / č. škola in Verbindung mit dt. gehen / č. jit/chodit bzw. dt. fahren / č. jet/jezdit zu durchsuchen. ${ }^{8}$ Aufgrund dieses Vorgehens sowie des größeren Umfangs der Korpora bilden im Vergleich zu den gesprochenen Korpora deutlich mehr Belege die Grundlage für diese Auswertung. Die Ergebnisse wurden insofern gefiltert, als dass nur jene mit den Präpositionen aus Tabelle 2 einfließen.

Abb. 5 gibt einen Überblick über die Präpositionswahl in den geschriebensprachlichen Korpora. Die Präposition dt. in / č. do kommt in direktionalen PPn mit dem Verb dt. gehen / č. jit/chodit im Tschechischen auf $84 \%$, im DiÖ auf $57 \%$, im DiD auf $32 \%$ und im Deutschen in der Schweiz auf $23 \%$. Dt. $z u /$ č. $k$ wird nur im deutschen Sprachraum verwendet, am häufigsten in der Schweiz in $73 \%$ der Fälle, gefolgt vom DiD mit $57 \%$ und dem DiÖ mit $35 \%$. Die Präpositionen dt. auf / č. na kommt nur im Tschechischen (16 \%) und im DiD (4\%) auf nennenswerte Anteile. Dt. an wird in allen drei

8 Durch die Abfrage zweier (hoch-)frequenter Bewegungsverben sollte ermöglicht werden, grob den potentiellen Einfluss intentionaler Kontexte (nach X gehen, um Y zu tun, z. B. Konstruktionen wie auf ein Bier gehen; vgl. Saicová Ř́malová 2009) einschätzen zu können. Wir gehen nämlich davon aus, dass dt. fahren / č. jet/jezdit deutlich seltener in solchen verwendet wird als dt. gehen / č. jit/chodit. Die Suchabfragen wurden für das DeReKo im Korpusmanager Cosmas II nach dem folgenden Muster gestaltet: (\&in / w +2 \&Schule) /s0 \&gehen. Das SYNv7 wurde im Korpusmanager Kontext mit Hilfe von nach der CQL-Syntax gestalteten Suchabfragen durchsucht: [lemma="jít|chodit"] []\{0,5\} [lemma="do"] [] $\{0,1\}$ [lemma="škola"] within <s $/>$ sowie [lemma= "do"] []\{0,1\} [lemma="škola"] []\{0,5\} [lemma="jít|chodit"] within <s $/>$. 


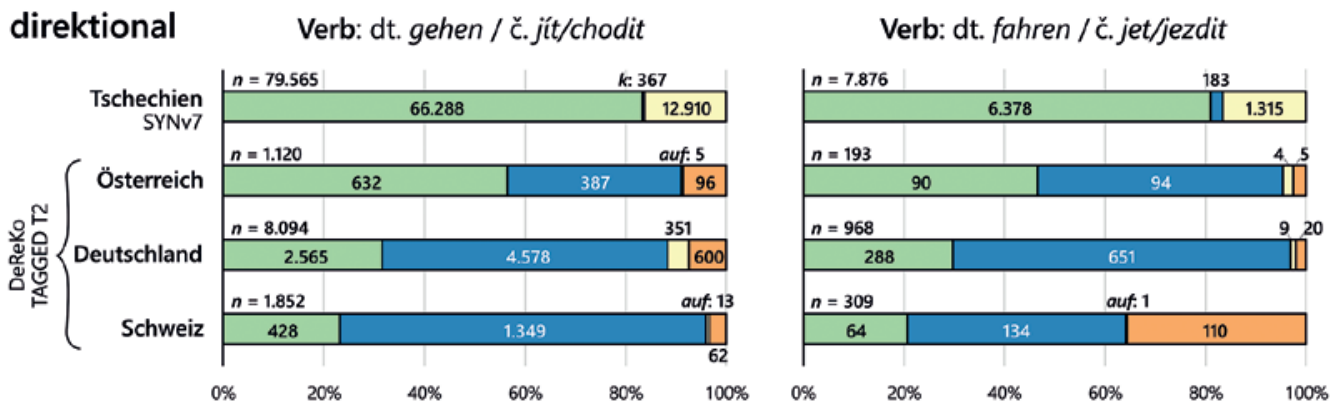

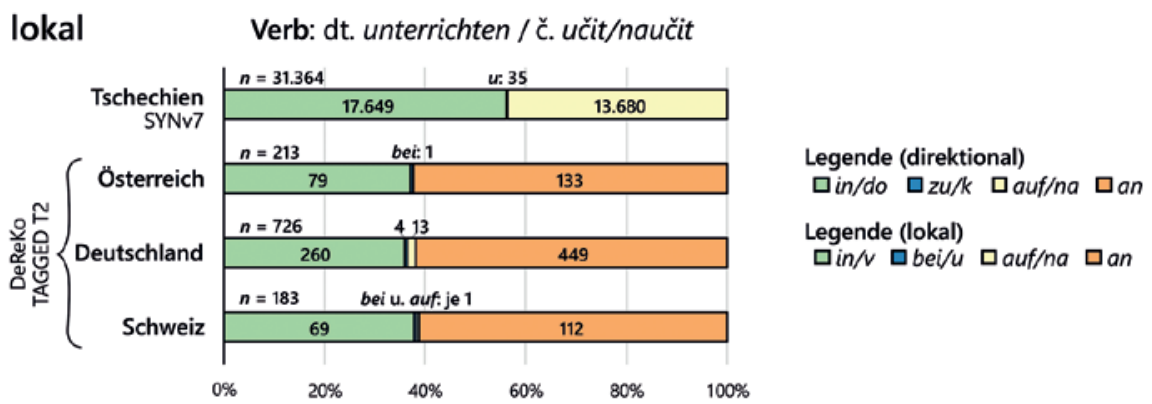

Abb. 5: Direktionale und lokale PPn in den geschriebensprachlichen Korpora - Überblick

deutschsprachigen Varietäten in $3 \%$ (Schweiz), $7 \%$ (DiD) bzw. $8 \%$ (DiÖ) der Belege gebraucht.

Die Präpositionswahl mit dem Verb dt. fahren / č. jet/jezdit zeigt für das Tschechische keine nennenswerten Unterschiede. Im DiÖ überwiegt die Präposition dt. $z u$ (47 \%) vor dt. in (45\%). Auch im DiD wird dt. $z u$ mit $70 \%$ deutlich häufiger verwendet, wohingegen dt. auf mit dt. fahren kaum gebraucht wird. Im Deutschen in der Schweiz zeigt sich hingegen ein völlig anderes Bild: So kommen dt. $z u$ in $44 \%$ (73\% bei dt. gehen) und dt. an in $36 \%$ der Fälle (3\% bei dt. gehen) zur Anwendung.

In lokalen PPn mit dem Verb dt. unterrichten / č. učit/naučit fällt die Präpositionswahl im Vergleich zu den direktionalen PPn höchst unterschiedlich aus. Im Tschechischen überwiegt zwar die Präposition č. $v$ (als lokale Entsprechung von č. do), wird jedoch in nur $56 \%$ der Fälle gebraucht. Die übrigen $44 \%$ entfallen auf č. na. Die Daten zu den drei deutschsprachigen Varietäten sind sehr homogen. Dt. in kommt auf rund $37 \%$, dt. an auf $63 \%$. Die Dominanz der Präposition dt. an ist in den geschriebensprachlichen Korpora des Deutschen noch deutlicher zu sehen als in den gesprochensprachlichen und erhärtet die Vermutung, dass die Präpositionswahl sowohl vom Nomen der PP als auch vom Verb abhängig ist.

Die deutschen schriftsprachlichen Daten können je nach Publikationsort der jeweiligen Zeitung verschiedenen geographischen Regionen zugeordnet werden, was eine diatopische Auswertung ermöglicht. Abb. 6 zeigt, dass die areale Variation an die Staatsgrenzen gebunden ist. Innerhalb des DiD gibt es im schriftsprachlichen, standardnahen 


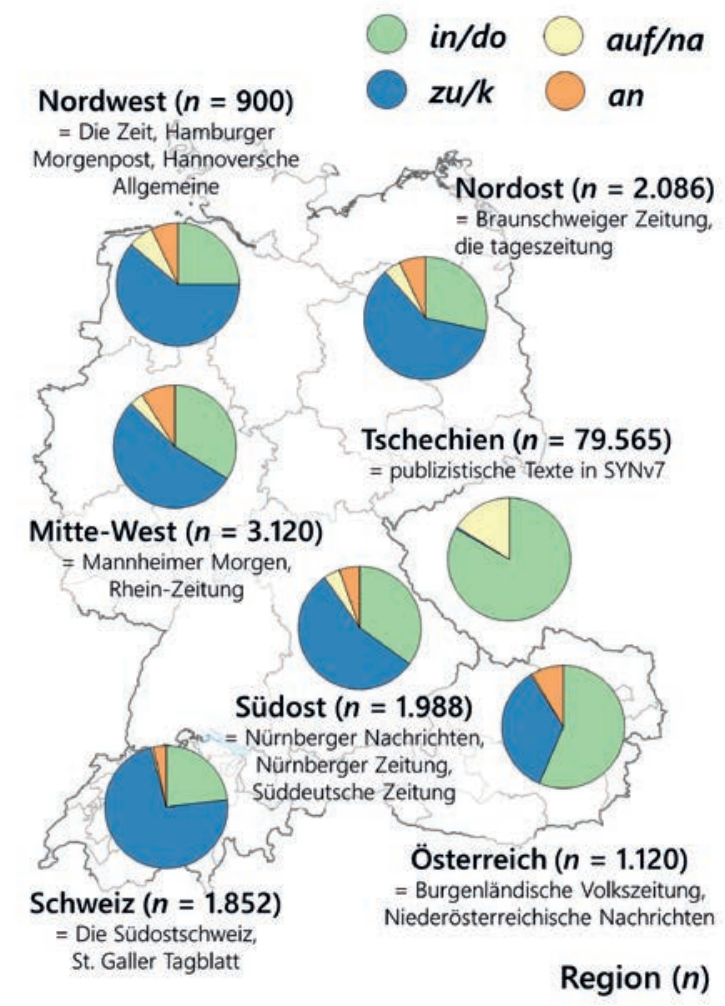

Abb. 6: Direktionale PPn mit dem Verb dt. gehen / č. jittchodit im arealen Überblick

Gebrauch kaum nennenswerte Unterschiede bei der Präpositionswahl mit dem Lemma dt. Schule; dt. $z u$ wird in allen Regionen in rund zwei Drittel der Fälle gebraucht, wobei die relative Frequenz von Nordwesten nach Südosten abnimmt. Für das Deutsche in der Schweiz zeigt sich eine etwas deutlichere Dominanz der Präposition dt. zu, für das DiÖ eine der Präposition dt. in, wobei angemerkt werden muss, dass das das durchsuchte Teilkorpus des DeReKo (TAGGED-2) nur Daten aus Ostösterreich (Niederösterreich, Burgenland) enthält.

Die morphosyntaktische Annotation der verwendeten Korpora macht diese einfach und schnell für rein quantitative Analysen durchsuchbar, birgt aber auch die Gefahr von falschen Treffern. Zwar wurden nicht-direktive Präpositionen nicht abgefragt, doch besteht auch bei den für diese Arbeit als direktiv definierten Präpositionen (vgl. Tabelle 2) die Möglichkeit eines nicht-direktiven Gebrauchs, der über die Suchabfrage allein nicht hinreichend ausgeschlossen werden kann. Zum Zwecke der Validierung der bis dato überblicksartigen Beschreibung der Präpositionswahl sowie vertiefender Annotationen wurde daher ein Subkorpus bestehend aus Zeitungstexten aus Tschechien, Österreich sowie Nordwestdeutschland (Hamburg und Hannover) erstellt (vgl. Abb. 7), welches manuell kontrolliert wurde. Die Häufigkeit der Verwendung der Präpositionen deckt 


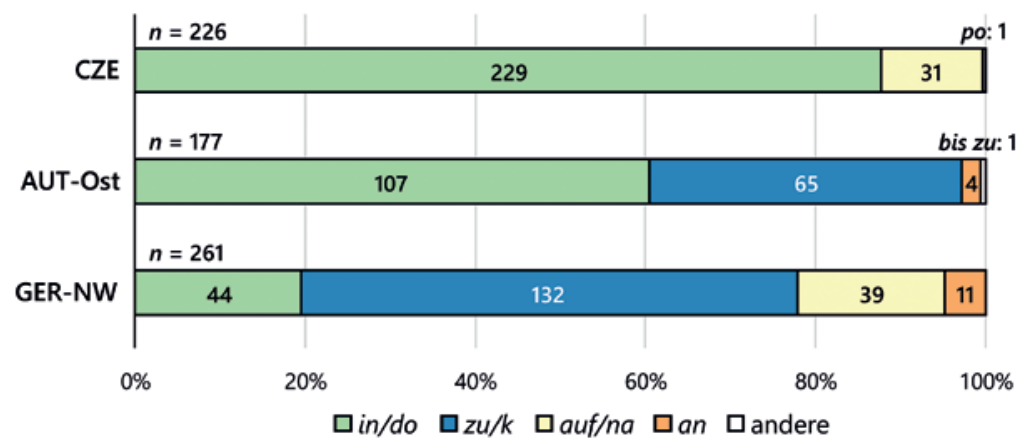

Abb. 7: Manuell annotierte Belege für direktive PPn

aus den geschriebensprachlichen Korpora

sich nach Aussortierung der falschen Treffer weiterhin gut mit jener des Überblickes mit einer Abweichung von max. $5 \%$, was die Aussagekraft der quantitativen Methode unterstreicht. Deutlich öfter wird nur im Subkorpus Nordwestdeutschlands die Präposition dt. auf gebraucht - $17 \%$ gegenüber den $4 \%$ in der Erstauswertung.

Auch dieses Subkorpus zeigt eine deutliche Differenz zwischen Präpositionswahl im (nordwestdeutschen) DiD und im DiÖ, darüber hinaus sowohl Überschneidungen zwischen dem Tschechischen und dem DiÖ (Häufigkeit von dt. in / č. do) als auch zwischen dem Tschechien und dem DiD (Häufigkeit dt. auf / ̌̌. na).

\section{Ausblick auf diachrone Variation}

Nachdem der Blick bis dato ein synchroner war, soll noch beispielhaft eine diachrone Perspektive auf das DiÖ eingenommen werden, um etwaige (Dis-)Kontinuitäten aufzuzeigen. Hierzu wurde ein kleines schriftsprachliches Korpus mit der Phrase Schule gehen mit in ANNO verfügbaren Zeitungsartikeln der Jahre $1778-1836$ aus Wien erstellt. ${ }^{9}$ Wie Abb. 8 zeigt, wird die Präposition dt. in in $85 \%$ der Fälle gebraucht - deutlich häufiger als die rund $60 \%$ im rezenten, schriftsprachlichen Korpus des DiÖ. Dt. auf kommt nur zwei Mal und dies in Verbindung mit höheren Schulen vor. Am bemerkenswertesten ist jedoch die geringe Häufigkeit der Präposition dt. $z u$, die im Gegensatz zu gegenwärtigen Zeitungstexten derselben Region (knapp $40 \%$ ) in nur $13 \%$ der Fälle verwendet wird. Die historischen relativen Frequenzen der Präpositionen dt. in und dt. $z u$ entsprechen damit tendenziell eher jenen, die für das rezente gesprochene DiÖ beschrieben werden konnten. Diese deutlichen Unterschiede in der Präpositionswahl zwischen dem rezenten und dem historischen Korpus des geschriebenen DiÖ sowie die Übereinstimmung des historischen geschriebenen Korpus mit dem rezenten gesprochenen können als erstes

9 Die Scans der Zeitungen und Zeitschriften wurden automatisch texterkannt und können im Volltext mit Hilfe von Abstandssuche und Wildcards sowie nach Metadaten durchsucht werden. Im vorliegenden Fall lautete die Suchanfrage „Schule gehen" 5 . 


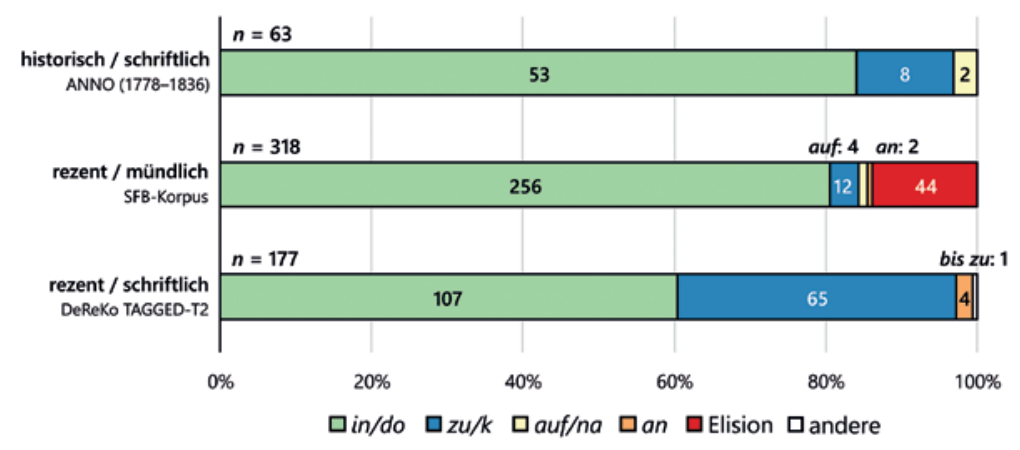

Abb. 8: Vergleich des historischen Korpus mit den rezenten Korpora des DiÖ in Bezug auf direktive PPn mit dem inneren Argument Schule

Indiz dafür gesehen werden, dass es sich bei der Verwendung von dt. $z u$ im geschriebenen, standardnahen DiÖ um eine Orientierung hin zum bundesdeutschen Standard handeln könnte.

\section{Ausblick auf semantische Faktoren für die Präpositionswahl}

Anschließend an die theoretischen Ausführungen zur Präpositionswahl in Abschnitt 2 stellt sich zudem die Frage, wie sich die beobachtete diatopische und diamediale Variation mit einem Modell wie jenem von Grießhaber (1999) in Einklang bringen lässt. Variiert die Klassifikation des inneren Arguments der PP in bestimmten Argumentklassen oder seine komplexe Relation zum äußeren Argument, sodass Schülerinnen und Schüler etwa in Österreich (und Tschechien) tendenziell eher in das Gebäude Schule hinein, jene in (Nordwest-)Deutschland jedoch nur in die Nähe des „Amtes“ Schule gehen? Oder variiert die Präposition, die zum Ausdruck der komplexen Relation in Bezug auf eine bestimmte Argumentklasse typischerweise selegiert wird? Zusätzlich ist zu berücksichtigen, dass die Wahl der Präposition auch stark in Verbindung mit dem konkreten inneren Argument der PP und nicht nur mit seiner Argumentklasse steht, wie ein Ausblick auf Überblicksdarstellungen mit anderen Lemmata verrät, die auf denselben Quellen wie die in Abschnitt 5 präsentierten basieren. Ein Lemma wie dt. Post / č. pošta, das ähnlich wie dt. Schule / č. škola laut Grießhaber (1999) sowohl als Raum/Bezirk als auch als Fläche/Amt klassifiziert werden könnte, zeigt auf den ersten Blick andere diatopische Variationsmuster in der geschriebenen Sprache: Während in direktiven Phrasen die Variante dt. zur Post gehen ebenso häufig ist wie dt. zur Schule gehen, ist in lokalen Kontexten nur dt. bei der Post arbeiten, nicht aber dt. *bei der Schule arbeiten geläufig. Umgekehrt kann man zwar dt. an der Schule unterrichten, nicht aber dt. *an der Post arbeiten. Auch im geschriebenen Tschechischen gibt es im Vergleich der beiden Lemmata große Unterschiede in der Präpositionswahl. So wird č. pošta sowohl in direktiven als auch lokalen Kontexten in 80-90 \% der Fälle mit č. na verwendet, č. škola in nur $8 \%$. 
Zudem zeigt der Vergleich der Variation bei dt. gehen / č. jit/chodit und dt. fahren / č. jet/jezdit, dass die Wahl der Präposition in direktiven Phrasen unter anderem vom Verb abhängt. Im geschriebenen DiÖ wird dt. zu mit dem ersten Verb etwa nur in $35 \%$, mit dem zweiten in knapp 50 \% der Fälle gebraucht. Im DiD und im Tschechischen sind die Schwankungen nicht so groß. Deutliche Unterschiede gibt es allerdings im Vergleich zu lokalen Phrasen mit dem Verb dt. unterrichten / č. učit/naučit, bei dem in den deutschen Varietäten die Präposition dt. an überwiegt und im Tschechischen der Anteil von č. na mit $44 \%$ deutlich über der dem entsprechenden Anteil bei direktiven Phrasen liegt. Semantische Einflussfaktoren, die über die bloße Klassifizierung des inneren Arguments und die komplexe Relation des inneren zum äußeren Argument hinausgehen, liegen daher nahe. Diesen Aspekt zu untersuchen, bleibt jedoch Folgestudien vorbehalten.

\section{Zusammenfassung}

Unsere hier vorliegenden Auswertungen haben gezeigt, dass die Präpositionswahl in direktiven Kontexten mit dem Lemma dt. Schule / č. škola als innerem Objekt einer PP sowohl diamedial als auch diatopisch variiert:

In allen drei untersuchten Sprachen bzw. Varietäten gibt es Unterschiede der Präpositionswahl zwischen gesprochener und geschriebener Sprache, wobei global betrachtet dt. in / ̌́. do in gesprochenen Varietäten häufiger auftritt als in geschriebenen. Im Folgenden werden die Detailergebnisse nochmals kurz nach staatsräumlich definierten Varietäten/Sprachen zusammengefasst:

- Im Tschechischen wird die Präposition č. $n a$ in direktiven Phrasen in geschriebener Form mit $16 \%$ deutlich häufiger gebraucht als in gesprochener mit $8 \%$. Die Präposition č. do überwiegt jedoch in beiden Korpora.

- Im DiÖ kommt die Präposition dt. in in gesprochener Form auf über $80 \%$, in geschriebener auf nur rund $55 \%$. Dt. $z u$ wird im gesprochenen DiÖ kaum verwendet, im geschriebenen allerdings - verbabhängig - in 35-50 \% der Fälle. Auch die Präposition dt. an (9\%) ist auf das geschriebene DiÖ beschränkt. Demgegenüber entfällt die Präposition nur im gesprochenen DiÖ, dies allerdings bei $14 \%$ der Belege.

- Im DiD sind die Unterschiede in der Präpositionswahl zwischen gesprochener und geschriebener Sprache nicht ganz so groß. Die Präposition dt. $z u$ wird in geschriebenen Form mit 55-65\% häufiger gebraucht als in gesprochener mit $37 \%$, bei dt. in ist die Verteilung umgekehrt (30 \% zu $50 \%$ ). Dt. an kommt im geschriebenen Korpus häufiger vor, dt. auf im gesprochenen, wobei der Anteil in beiden Fällen bei max. $7 \%$ liegt.

Bezogen auf diatopische Variation lassen sich sowohl sprachübergreifende areale Muster als auch regionale Spezifika identifizieren. Zunächst finden wir im deutschen Sprachraum eine deutliche Nord(west)-Süd(ost)-Gliederung, wobei im Nordwesten die Präposition dt. $z u$, im Südosten - und damit Österreich, aber graduell auch in Bayern - hingegen dt. in überwiegt. Erste diachrone Indizien zeigen, dass die nordwestliche Variante auch im südöstlichen Gebiet progressiv ist. In diese Gliederung fügt sich das 
Tschechische insofern mit ein, als es die zu dt. in quasiäquivalente Präposition č. do präferiert, das Quasiäquivalent zu dt. zu, nämlich č. $k$ hingegen gar nicht kennt. Die dominante Präposition č. do variiert nur mit č. na, dessen deutsche Quasiäquivalente dt. auf und an zumindest in den gesprochenen Varietäten insbesondere im mittel(ost) deutschen Raum auftreten. Im gesprochenen tschechischen Korpus ergibt eine erste Auswertung interessanterweise einen regionalen Fokus der $n a$-Variante auf den benachbarten nord- und zentralböhmischen Raum.

Wie sich die Schweiz mit ihrer starken diamedialen Variation in dieses areale Bild einfügt, muss in künftigen Studien genauer untersucht werden. Im besten Fall sollten diese auch weitere Sprachen rund um die hier untersuchten, also etwa das Niederländische, Polnische, Slowakische, Slowenische und Ungarische ${ }^{10}$ berücksichtigen. ${ }^{11}$

Der vorliegende Artikel konnte nachweisen, dass bei der Beschreibung der Präpositionswahl in direktiven und lokalen Präpositionalargumenten in zentraleuropäischen Sprachen auch sprachübergreifend Aspekte diatopischer (arealer) und diamedialer Variation berücksichtigt werden müssen. In weiteren Schritten sind diachrone Variationsmuster zu beschreiben sowie semantische Faktoren für die Präpositionswahl zu identifizieren.

\section{Korpora}

Institut für Deutsche Sprache (IDS) (2014): Deutsches Referenzkorpus / Archiv der Korpora geschriebener Gegenwartssprache 2014-I (Release vom 15. 4. 2014). Mannheim: Institut für Deutsche Sprache. Online verfügbar unter www.ids-mannheim.de/DeReKo, zuletzt geprüft am 27. 12. 2020.

Institut für Deutsche Sprache (IDS) (2015): Datenbank für Gesprochenes Deutsch (DGD). Deutsche Umgangssprachen: Pfeffer-Korpus. Online verfügbar unter http://dgd.ids-mannheim.de, zuletzt geprüft am 27. 12. 2020.

Institut für Deutsche Sprache (IDS) (2021): Datenbank für Gesprochenes Deutsch (DGD). Deutsch Heute. Version 2.15. Online verfügbar unter http://dgd.ids-mannheim.de, zuletzt geprüft am 25. 6. 2021.Kopřivová, Marie [et. al.] (2017): ORTOFON: Korpus neformální mluvené češtiny s víceúrovňovým přepisem. Praha: Ústav Českého národního korpusu FF UK. Online verfügbar unter http:/ / www.korpus.cz, zuletzt geprüft am 27. 12. 2020.

Křen, Michal [et. al.] (2015): SYN2015: reprezentativní korpus psané češtiny. Praha: Ústav Českého národního korpusu FF UK. Online zugänglich unter http://www.korpus.cz, zuletzt geprüft am 10. 1. 2021.

Křen, Michal [et. al.] (2018): Korpus SYN, verze 7 z 29. 11. 2018. Praha: Ústav Českého národního korpusu FF UK. Online verfügbar unter http://www.korpus.cz, zuletzt geprüft am 10. 1. 2021.

10 Im Ungarischen wären statt der Präpositionen die entsprechenden Suffixmorpheme zu untersuchen.

11 Es ist davon auszugehen, dass die Berücksichtigung romanischer Sprachen wie des Französischen und Italienischen wenig ergiebig ist, da diese die Wegkomponenten (Ausgangspunkt, Weg und Ziel) einer Bewegung nicht in Argumenten des Verbs, sondern am Verb selbst ausdrücken (vgl. die Unterscheidung von verb-framed languages [z. B. der romanischen Sprachen] von satellite-framed languages [wie den im Text genannten] bei Talmy 2000: 49-57). Die Präpositionen sind also auch in Argumenten von Bewegungsverben auf ihre syntaktische Funktion reduziert. 


\section{Wörterbücher und andere Nachschlagewerke}

Ammon, Ulrich - Bickel, Hans - Lenz, Alexandra N. (hrsg.) (2016): Variantenwörterbuch des Deutschen. Die Standardsprache in Österreich, der Schweiz, Deutschland, Liechtenstein, Luxemburg, Ostbelgien und Südtirol sowie Rumänien, Namibia und Mennonitensiedlungen. 2., völlig neu bearbeitete und erweiterte Auflage. Berlin - Boston: de Gruyter.

Dürscheid, Christa - Elspaß, Stephan - Ziegler, Arne (hrsg.) (2018): Variantengrammatik des Standarddeutschen. Ein Online-Nachschlagewerk. Online verfügbar unter http://mediawiki. ids-mannheim.de/VarGra/index.php/Start, zuletzt geprüft am 27. 12. 2020.

Ebner, Jakob (2009): Duden. Wie sagt man in Österreich? Wörterbuch des Österreichischen Deutsch. 4., völlig überarbeitete Auflage. Berlin: Dudenverlag.

Elmentaler, Michael - Rosenberg, Peter (2015): Norddeutscher Sprachatlas (NOSA) 1. Regiolektale sprachlagen. Hildesheim [u. a.]: Olms.

Elspaß, Stephan - Möller, Robert (2003ff.): Atlas zur deutschen Alltagssprache (AdA). Online verfügbar unter http://www.atlas-alltagssprache.de/, zuletzt geprüft am 27. 12. 2020.

Kim, Agnes (2021): Phänomengruppen im Überblick: Von Morphonologie bis Syntax. In Kim, Agnes - Newerkla, Stefan Michael (hrsg.): MiÖ-SAKON - Sprachliche Areal- und Kontaktphänomene im Deutschen in Österreich. Online verfügbar unter: https://wiki.dioe.at/books/ was-ist-mi\%C3\%B6-sakon-eine-einf\%C3\%BChrung/page/ph\%C3\%A4nomengruppen-im\%C3\%BCberblick-von-morphonologie-bis-syntax, zuletzt geprüft am 28. 01. 2021.

Kleiner, Stefan (2011-2021): Atlas zur Aussprache des deutschen Gebrauchsstandards (AADG). Unter Mitarbeit von Ralf Knöbl. Online verfügbar unter: http://prowiki.ids-mannheim.de/ bin/view/AADG/, zuletzt geprüft am 25. 6. 2021.

Kloferová, Stanislava (2017): NÁŘEČNí SKUPINA (makrodialekt). In: Karlík, Petr - Nekula, Marek - Pleskalová, Jana (hrsg): CzechEncy - Nový encyklopedický slovník češtiny. Online verfügbar unter https://www.czechency.org/slovnik/NÁŘEČNÍ SKUPINA, zuletzt geprüft am 10. 1. 2021.

Wöllstein, Angelika - Dudenredaktion (hrsg.) (2016): Duden. Die Grammatik. Unentbehrlich für richtiges Deutsch. 9., vollständig überarbeitete und aktualisierte Auflage. Berlin: Dudenverlag.

Zifonun, Gisela - Hoffmann, Ludger - Strecker, Bruno (1997): Grammatik der deutschen Sprache. Berlin - New York: de Gruyter.

\section{Literatur}

Bednarský, Petr (2002): Deutsche und tschechische Präpositionen kontrastiv. Am Beispiel von an, auf und $n a$. Münster: Waxmann.

De Cillia, Rudolf - Ransmayr, Jutta (2019): Österreichisches Deutsch macht Schule. Bildung und Deutschunterricht im Spannungsfeld von sprachlicher Variation und Norm. Unter Mitarbeit von Ilona Elisabeth Fink. Wien - Köln - Weimar: Böhlau.

Ebner, Jakob (1988): Wörter und Wendungen des österreichischen Deutsch. In: Wiesinger, Peter (hrsg.): Das Österreichische Deutsch. Wien - Köln - Graz: Böhlau, S. 99-187.

Ebner, Jakob (2008): Duden. Österreichisches Deutsch. Eine Einführung. Mannheim [et. al.]: Dudenverlag.

Elmentaler, Michael [et. al] (2015): Sprachvariation in Norddeutschland (SiN). In: Kehrein, Roland - Lameli, Alfred - Rabanus, Stefan (hrsg): Regionale Variation des Deutschen. Projekte und Perspektiven. Berlin [et. al.]: De Gruyer Mouton, S. 397-424. 
Ganswindt, Brigitte - Kehrein, Roland - Lameli, Alfred (2015): Regionalsprache.de (REDE). In: Kehrein, Roland - Lameli, Alfred - Rabanus, Stefan (hrsg): Regionale Variation des Deutschen. Projekte und Perspektiven. Berlin [et. al.]: De Gruyer Mouton, S. 425-457.

Grießhaber, Wilhelm (1999): Die relationierende Prozedur. Zu Grammatik und Pragmatik lokaler Präpositionen und ihrer Verwendung durch türkische Deutschlerner. Zugl.: Hamburg, Univ., Habil.-Schr., 1991. Münster: Waxmann (Mehrsprachigkeit 5).

Grießhaber, Wilhelm (2007): Präposition. In: Hoffmann, Ludger (hrsg.): Handbuch der deutschen Wortarten. Berlin - New York: de Gruyter, S. 629-655.

Hnátková, Milena [et. al.] (2014): The SYN-series corpora of written Czech. In: Calzolari, Nicoletta (hrsg.): Proceedings of the Ninth International Conference on Language Resources and Evaluation (LREC 2014), Reykjavik, Iceland, May 26-31, 2014. European Language Resources Association (ELRA), S. 160-164.

Hundt, Markus (2001): Grammatikalisierungsphänomene bei Präpositionalobjekten in der deutschen Sprache. In: Zeitschrift für Germanistische Linguistik 29, S. 167-191.

Kim, Agnes (2020): Prepositions in the Melting Pot: High Risk of Infection. Language Contact of German in Austria with Slavic Languages and its Linguistic and Extra-Linguistic Description. In: Szucsich, Luka - Kim, Agnes - Yazhinova, Uliana (hrsg.): Areal Convergence in Eastern Central European Languages and Beyond. Berlin [et. al.]: Peter Lang, S. 95-137.

Kim, Agnes - Scharf, Sebastian - Šimko, Ivan (2020): Variation in Case Government of the Equivalent for the Cogntive Verb to forget in German in Austria and Czech. In Szucsich, Luka - Kim, Agnes - Yazhinova, Uliana (hrsg.): Areal Convergence in Eastern Central European Languages and Beyond. Berlin [et. al.]: Peter Lang, S. 139-175.

Kleiner, Stefan (2015): „Deutsch heute“ und der Atlas zur Aussprache des deutschen Gebrauchsstandards. In: Kehrein, Roland - Lameli, Alfred - Rabanus, Stefan (hrsg): Regionale Variation des Deutschen. Projekte und Perspektiven. Berlin [et. al.]: De Gruyer Mouton, S. 489-518.

Komrsková, Zuzana [et. al.] (2017): New Spoken Corpora of Czech: ORTOFON and DIALEKT. In: Journal of Linguistics/Jazykovedný casopis 68, S. 219-228.

Koppensteiner, Wolfgang - Lenz, Alexandra N. (2017): Theoretische und methodische Herausforderungen einer perzeptiv-attitudinalen Standardsprachforschung. Perspektiven aus und auf Österreich. In: Sieburg, Heinz - Solms, Hans-Joachim (hrsg.): Das Deutsche als plurizentrische Sprache. Ansprüche - Ergebnisse - Perspektiven. Berlin: Erich Schmidt Verlag (= Zeitschrift für deutsche Philologie 136), S. 43-68.

Křen, Michal [et. al.] (2016): SYN2015: Representative Corpus of Contemporary Written Czech. In: Nicoletta Calzolari (hrsg.): LREC 2016, Tenth International Conference on Language Resources and Evaluation. May 23-28, 2016, Grand Hotel Bernardin Conference Center, Portorož, Slovenia. Paris: European Language Resources Association, S. 2522-2528.

Lenz, Alexandra N. [et. al.] (2019): Exploring syntactic variation by means of "Language Production Experiments": Methods from and analyses on German in Austria. In: Journal of Linguistic Geography 7, S. 63-81.

Leys, Odo (1989): Aspekt und Rektion räumlicher Präpositionen. In: Deutsche Sprache. Zeitschrift für Theorie, Praxis und Dokumentation 17, S. 97-113.

Masařík, Zdeněk (1998): Zum tschechisch-deutschen/österreichischen Sprachkontakt (dargestellt am Beispiel Südmähren - Niederösterreich). In: Bauer, Werner - Scheuringer, Hermann (Hg.): Beharrsamkeit und Wandel. Festschrift für Herbert Tatzreiter zum 60. Geburtstag. Wien: Praesens, S. 133-141.

Muhr, Rudolf (1995): Grammatische und pragmatische Merkmale des Österreichischen Deutsch. In: Muhr, Rudolf - Schrodt, Richard - Wiesinger, Peter (hrsg.): Österreichisches Deutsch. 
Linguistische, sozialpsychologische und sprachpolitische Aspekte einer nationalen Variante des Deutschen. Wien: hpt, S. 208-235.

Newerkla, Stefan Michael (2007): Areály jazykového kontaktu ve střední Evropě a německo-český mikorareál ve východním Rakousku. In: Slovo a slovesnost 68, S. 271-286.

Newerkla, Stefan Michael (2009): Bohemismen (und Slowakismen) in Wien (und Österreich). In: tribüne. zeitschrift für sprache und schreibung, Heft 3, S. 8-12.

Newerkla, Stefan Michael (2013): Linguistic Consequences of Slavic Migration to Vienna in the 19th and 20th Centuries. In: Moser, Michael - Polinsky, Maria (hrsg.): Slavic languages in migration. Berlin [et. al.]: LIT, S. 247-260.

Newerkla, Stefan Michael (2017): Kontaktareale in Mitteleuropa am Beispiel Altösterreich. In: Mauerer, Christoph (hrsg.): Mehrsprachigkeit in Mittel-, Ost- und Südosteuropa. Gewachsene historische Vielfalt oder belastendes Erbe. Beiträge zur 1. Jahrestagung des Forschungszentrums Deutsch in Mittel-, Ost- und Südosteuropa, Regensburg, 2.-4. Oktober 2014 (= Forschungen zur deutschen Sprache in Mittel-, Ost- und Südosteuropa FzDiMOS Band 4). Regensburg: Verlag Friedrich Pustet, S. 17-32. [https://phaidra.univie.ac.at/o:961978]

Pfeffer, Jay Alan (1975): Grunddeutsch. Erarbeitung und Wertung dreier deutscher Korpora. Ein Bericht aus dem „Institute for Basic German“, Pittsburgh. Tübingen: Narr.

Saicová Ř́malová, Lucie (2009): O významech sloves typu jít a typu chodit v češtině. (Pohled kognitivní). In: Bohemistyka (3), S. 161-176.

Schuchardt, Hugo (1884): Slawo-Deutsches und Slawo-Italienisches. Dem Herrn Franz von Miklosich zum 20. 11. 1883. Graz: Leuschner und Lubensky.

Talmy, Leonard (2000): Toward a cognitive semantics. Volume I: Concept structuring systems. Cambridge, Mass: MIT Press.

Wiesinger, Peter (1996): Das österreichische Deutsch als eine Varietät der deutschen Sprache. In: Die Unterrichtspraxis 29, S. 154-164.

Zeman, Dalibor (2003): Das österreichische Deutsch und die österreichisch-tschechischen Sprachbeziehungen. Ein kulturhistorischer und sprachlicher Abriß. Dissertation. Universität Wien.

MMag. Agnes Kim / agnes.kim@univie.ac.at

Universität Wien, Institut für Slawistik

Spitalgasse 2, Hof 3, 1090 Wien, AT

Maria Schinko, BA BA MA MA / maria.schinko@univie.ac.at

Universität Wien, Institut für Slawistik

Spitalgasse 2, Hof 3, 1090 Wien, AT

This work can be used in accordance with the Creative Commons BY-SA 4.0 International license terms and conditions (https://creativecommons.org/licenses/by-sa/4.0/legalcode). This does not apply to works or elements (such as image or photographs) that are used in the work under a contractual license or exception or limitation to relevant rights 